\title{
A New Location Sensing Algorithm Based on DV-Hop and Quantum- Behaved Particle Swarm Optimization in WSN
}

\author{
Dan Zhang ${ }^{1, *}\left({ }^{*}\right.$, Xiaohuan Zhang $^{1}$ and Hai $\mathrm{Qi}^{2}$ \\ ${ }^{1}$ School of Computer Science and Engineering, Huizhou University, Huizhou 516007, China \\ ${ }^{2}$ Network and Information Centre, Huizhou University, Huizhou 516007, China \\ * Corresponding author: Dan Zhang (zhangdan@hzu.edu.cn)
}

Manuscript Revised 23 May 2021; Accepted 29 May 2021; Published 29 May 2021

Academic Editor: Weiwei Cai $\mathbb{D}$

Abstract: In wireless sensor network, the location sensing of the sensor nodes is practical. If there is no location information of the sensor nodes, the perceived data would have no meaning. In recent years, the range-free location sensing algorithms have got great attention. DV-Hop localization algorithm is one of the important algorithm in rangefree location algorithms. It has high efficiency, convenient operation and low energy consumption. However, the localization accuracy cannot meet the requirements in some applications. In this paper, a new localization method is proposed, which is based on DV-Hop and Quantum-behaved Particle Swarm Optimization (QPSO) algorithm. First, it deals with the high influence of average single jumping distance and then modifies the calculation of it in the DV-Hop algorithm. Second, in order to solve the problem of the coordinate optimization in the DV-Hop algorithm, this study chooses QPSO algorithm to optimize the unknown nodes' coordinates. Simulation results show that the new method can improve the localization accuracy of the unknown nodes obviously in WSN.

Index Terms: Localization Sensing,DV-Hop Algorithm,Average-Hop Distance, QPSO, Localization Error Rate.

\section{Introduction}

Wireless sensor network (Wireless Sensor Networks, WSN) consists of a large number of sensor nodes, the sensor nodes are in self-organized and multi hop way that constitute a wireless network. They can perceive, acquire and process the information. The WSN connects to the external network (Internet/Satellite Network/Mobile Communication Network) via the sink node, and then transmits the information to the terminal equipment. Among them, the sink node is a node that the capabilities of storage, processing and communication are strong. The schematic diagram of the wireless sensor network is shown in figure 1 .

The characteristics of WSN can be summarized into two categories: one is the characteristics of sensors, and the other is the characteristics of the network. In WSN, it consists of a large number of micro sensor nodes and the nodes are energy-constrained and they have low-power dissipation.
Most of sensor nodes include four parts: the sensing module, the processor module, the wireless communication module

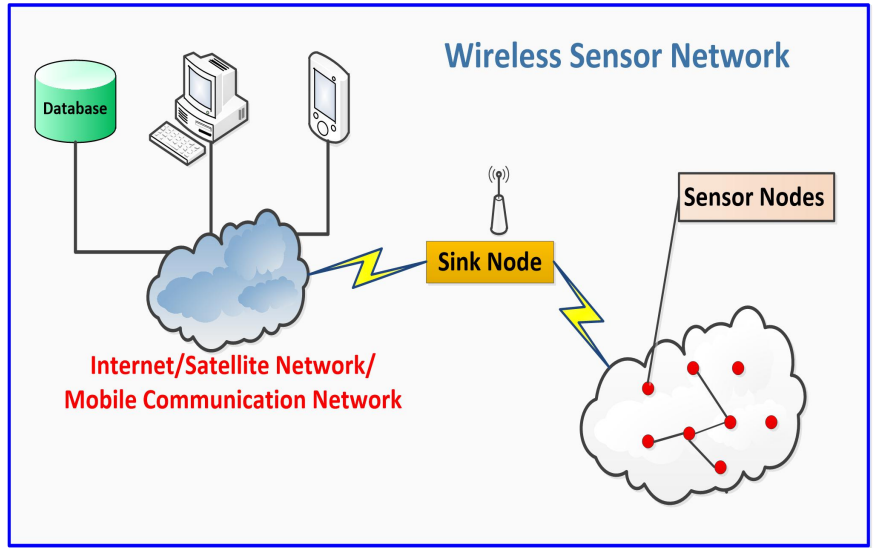

Fig.1. Schematic Diagram of Wireless Sensor Network.

and the power management module. However, due to the sensor nodes are very small, they are usually limited in energy and cannot operate independently for a long time. Moreover, the energy of the sensor nodes usually cannot be 
supplemented at any time because of the environmental factors. In addition, the computing power and data storage capability of the node are limited. Based on the characteristics of sensor nodes, the operation mode of the whole network is self-organizing. The network is similar to the Ad Hoc network. The communication radius of the nodes are small and the bandwidth is not high. The topology is relatively stable, strong dynamic, re-configurable and selfadjustment. There are two kinds of sensor nodes in WSN, one kind is called anchor nodes which can locate their location with hardware such as GPS and etc., and the other kind is called unknown nodes which cannot locate their location. The sensor nodes in wireless sensor network are usually distributed randomly in a designated region. Since the anchor nodes' localization requires additional equipment, the cost is higher. Therefore, most of the sensor nodes are unknown nodes in WSN. How to determine the locations of the unknown nodes is one of the key issues in WSN [1-2]. In this paper, we propose a new location sensing algorithm named SQPSODV-Hop (Standard Quantum Particle Swarm Optimization DV-Hop) localization algorithm. SQPSODVHop localization algorithm is based on DV-Hop and QPSO algorithm. It is from the average hop distance correction and estimated coordinates optimization of the unknown nodes to increase the accuracy of the unknown nodes.

The remainder of this paper is organized as follows: In Section 2, we introduce the related work about DV-Hop and QPSO algorithm. In Section 3, we analyze the main reason of producing error in DV-Hop location sensing algorithm. In Section 4, we propose SQPSODV-Hop localization sensing algorithm. In Section 5, it is the analysis of communication overhead and computational complexity of SQPSODV-HOP algorithm. In Section 6, we analyze iterative trends comparison of PSO and QPSO algorithm. In Section 7, we review performance comparison of proposed localization sensing algorithm at various simulation parameters. In section 8 we present the conclusions of this work.

\section{Related Work}

In this Section, we discuss the categories of location sensing algorithms and list several typical location sensing algorithms. Finally we focus on the DV-Hop and QPSO (Quantum-behaved Particle Swarm Optimization) algorithm.
In order to solve the coordination optimization problem of DV-Hop algorithm, QPSO algorithm is selected to optimize the coordinates of unknown nodes. The traditional trilateral measurement method and maximum likelihood estimation method have large errors in the calculation results, so in this section, QPSO algorithm is used to optimize the coordinates of the unknown nodes to reduce the positioning error. Compared with differential particle swarm optimization (DPSO), QPSO can avoid the problem of local optimal value in the process of location optimization, improve the accuracy of location without increasing communication overhead and computational complexity, reduce the number of iterations, and quickly enter the global convergence state.

\subsection{The Categories of Location Sensing Algorithms}

In wireless sensor network, the location sensing algorithms are usually divided into two categories: rangebased algorithm and range-free algorithm. The range-based localization algorithm uses additional hardware and measures distance from neighbor nodes. However, the range-free localization algorithm doesn't need them.

\section{(1) Range-based location sensing algorithm}

Range-based location sensing algorithm includes RSSI(Received Signal Strength Indication), and it is based on the intensity of the arriving signal algorithm, AOA algorithm (Angle of Arrival), TOA algorithm (Time of Arrival) and TDOA algorithm (Time Difference of Arrival) and so on $[3-4]$.

In the above algorithms, when the distance information of more than three anchor nodes is obtained by range-based location sensing algorithm, the location information of unknown nodes can be obtained through the mapping between distance and position, and the three side measurement is one of the commonly used methods. The following are the principles of trilateration. It is a commonly used method. Suppose that $P(\mathrm{x}, \mathrm{y})$ is an unknown node, $A\left(\mathrm{x}_{a}, \mathrm{y}_{a}\right), B\left(\mathrm{x}_{b}, \mathrm{y}_{b}\right)$ and $C\left(\mathrm{x}_{c}, \mathrm{y}_{c}\right)$ are the three anchor nodes, and the distances from $P$ to $A$ is $d_{a}$, the distances from $P$ to $B$ is $d_{b}$, the distances from $P$ to $C$ is $d_{c}$, as is shown in figure 2 . The relation between node $P$ and $A, B, C$ can be expressed in formula (1). 


$$
\left\{\begin{array}{l}
\sqrt{\left(x-x_{a}\right)^{2}+\left(y-y_{a}\right)^{2}}=d_{a} \\
\sqrt{\left(x-x_{b}\right)^{2}+\left(y-y_{b}\right)^{2}}=d_{b} \\
\sqrt{\left(x-x_{c}\right)^{2}+\left(y-y_{c}\right)^{2}}=d_{c}
\end{array}\right.
$$

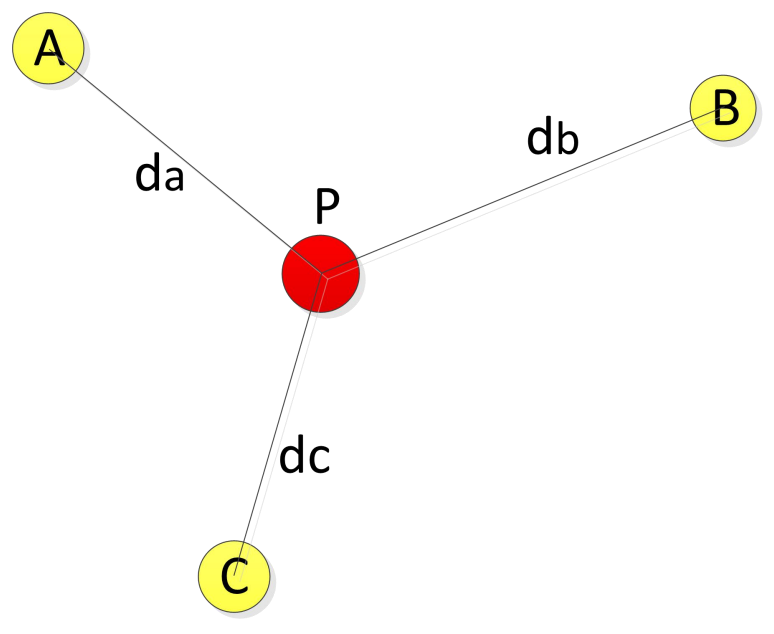

Fig 2. the trilateration in range-based location algorithm

According to the formula (1), the coordinates of the unknown nodes can be calculated. The computation use the following formula (2).

$$
\left[\begin{array}{l}
x \\
y
\end{array}\right]=\left[\begin{array}{ll}
2\left(x_{a}-x_{c}\right) & 2\left(y_{a}-y_{c}\right) \\
2\left(x_{b}-x_{c}\right) & 2\left(y_{a}-y_{c}\right)
\end{array}\right]^{-1}\left[\begin{array}{l}
x_{a}^{2}-x_{c}^{2}+y_{a}^{2}-y_{c}^{2}+d_{c}^{2}-d_{a}^{2} \\
x_{b}^{2}-x_{c}^{2}+y_{b}^{2}-y_{c}^{2}+d_{c}^{2}-d_{b}^{2}
\end{array}\right]
$$

Range-based location sensing algorithm has higher localization accuracy compared with range-free location sensing algorithm. Although range-based location sensing algorithm can get more accurate location results, it needs additional hardware equipment to measure distance and angle information. So these algorithms are not suitable for application in low-cost application.

\section{(2) Range-free location sensing algorithm}

Compared with range-based location sensing algorithm, range-free location sensing algorithm is more suitable in WSN with large-scale. It is low cost and estimates the location by using network topology and the information among nodes. Range-free location sensing algorithm depends on connectivity information to get the location information. They have got widespread concern in recent years [5-7]. The connectivity information of network mainly includes neighbor node information, hop value and average hop distance, and the mapping relation between the connectivity information and distance is analyzed below. It is assumed that there are two anchor nodes, they are anchor nodes $A_{i}$ and $A_{j}$, whose coordinates are $A_{i}\left(x_{i}, y_{i}\right)$ and $A_{j}\left(x_{j}, y_{j}\right)$ respectively, and the information of $A_{i}$ and $A_{j}$ are the location information of the neighbor nodes of unknown nodes. $h_{i j}$ is used to represent the hop values between anchor nodes $A_{i}$ and $A_{j}, H_{o p S i z e}$ is the average hop distance, and $A$ is the set of all anchor nodes. The average hop distance is calculated and broadcasted in wireless sensor networks. In order to obtain the location of unknown nodes, the average hop distance of the first received should be recorded, and the average hop distance is forwarded to the other unknown neighbor nodes. When the unknown nodes obtain the average hop distance, the distance between the anchor node and the unknown node is calculated according to the distance obtained from the first step. Thus, we can get the mapping relation of neighborhood information, hop value and average hop distance in the network. This mapping method is the basic method for estimating the distance between nodes in range-free localization sensing algorithms. The mapping relation between connectivity information and distance is shown in formulas (3) and (4).

$$
\begin{gathered}
\text { HopSize } i=\frac{\sum_{i \neq j, i, j \in A} \sqrt{\left(x_{i}-x_{j}\right)^{2}+\left(y_{i}-y_{j}\right)^{2}}}{\sum_{i \neq j, i, j \in A} h_{i j}} \\
d_{i j}=\text { HopSize }_{i} \times h_{i j}
\end{gathered}
$$

Range-free location sensing algorithm includes Centroid algorithm [8-9], APIT algorithm [10-12], DV-Hop algorithm[13-14] and Amorphous algorithm. But the location accuracy of the range-free location sensing algorithm is relatively low, and it needs to be improved.

\subsection{DV-Hop Algorithm}

DV-Hop location sensing algorithm is one of the important range free algorithms in WSN. It is proposed by Dragos Niculescu etc[15]. The advantages of DV-Hop location sensing algorithm are as follows: no additional hardware, simplicity in operation and low energy consumption. In the DV-Hop algorithm, the distance between the unknown node and the anchor nodes is estimated by distance vector and the number of hops, and 
then the location information of the unknown nodes is calculated by three-sided measurement or maximum likelihood estimation [16].

The implementation steps of DV-Hop algorithm are as follows [17-18]:

Step 1: Each anchor node broadcasts a packet to its neighbor nodes, the packet consists of the nodes' id, position and the hop count value (the initial value is 0 ). Each anchor node receives the packet and records the minimal hop count from the same anchor nodes. Then it forwards the packet to its neighbor nodes. Through this process, all the nodes will get the minimum hop count value from each anchor node.

Step 2: The average hop distance is calculated by using the following formula (5).

$$
\text { HopSize }_{i}=\frac{\sum_{i \neq j} \sqrt{\left(x_{i}-x_{j}\right)^{2}+\left(y_{i}-y_{j}\right)^{2}}}{\sum_{i \neq j} h_{i j}}
$$

In formula (5), HopSize ${ }_{i}$ is the average hop distance, $\left(x_{i}, y_{i}\right)$ and $\left(x_{j}, y_{j}\right)$ are the coordinates of the anchor nodes $i, j, h_{i j}$ is the hop count value between node $i$ and $j$. The anchor nodes broadcast the average hop distance to the network with a lifetime field. The first received average hop distance (as a correct value) by the unknown node is recorded, and forwards it to the neighbor nodes. This ensures that the correct value received by the node is sent by the nearest anchor node. After the process the unknown nodes calculate the distance from each anchor node. The distance can be estimated by formula (6).

$$
d_{i j}=\text {HopSize}_{i} * h_{i j}
$$

Step 3: When the unknown node obtains the distance from at least three anchor nodes, it calculates the coordinates of the unknown nodes by using the trilateration method or maximum likelihood estimation method.

In the literature [19], it was proposed that the hop values between anchor nodes and unknown nodes were corrected by using the RSSI value of the received packets. This idea can more accurately calculate the distance between sensor nodes in the DV-Hop localization algorithm.

In the literature [20], the average hop distance in the DVHop localization algorithm was corrected by the error correction value of $\delta$, which reduces the deviation between the estimated average hop distance and the actual average hop distance. The improved algorithm effectively reduces the average location error of unknown nodes and improves the location accuracy.

In the literature [21], the method of WLS (Weighted Least Squares) was used to refine the coordinates of the unknown nodes, and more accurate information was obtained by using WLS to solve the equations.

In the literature [22], a weighted DV-Hop localization algorithm for wireless sensor networks based on correction factor was proposed on the basis of selecting reference anchor nodes and computing the average hop distance of unknown nodes. The average hop distance for unknown node was based on the information of all anchor nodes within a range of hops.

In the literature [23], the author analyzed the relationship between the error of DV-Hop localization algorithm and the linearity. In this paper, an anchor node selection algorithm that meets the threshold and linear conditions of hop values is proposed to participate in the localization process. Since it is difficult to determine whether an anchor node is within one hop range, the Voronoi (Tyson polygon) graph was used to partition the sensor network into several regions. It can get anchor node information in each Voronoi polygon area. The algorithm takes advantage of these information and linear conditions to estimate the location of unknown nodes.

In the literature [24], the authors proposed a DDV-hop (Differential DV-hop) algorithm. In this algorithm, each anchor $A_{i}$ not only broadcasts its average hop distance, but also broadcasts the differential error of the average hop distance to the entire network. This differential error denoted as diff_error ${ }_{i}$, it is calculated by formula (7). Where $m_{d}$ is the number of anchors, hop $p_{i, j}$ is the hop count between $A_{i}$ and every other anchor $A_{j}, d_{i, j}$ is the distance between $A_{i}$ and $A_{j}$.

$$
\text { diff_error }_{i}=\frac{\sum_{j \neq i}\left|d p h_{i}-\frac{d_{i, j}}{h o p_{i, j}}\right|}{m_{d}-1}
$$

In the literature [25], the authors proposed a new localization algorithm based on TDOA and PSO. The 
algorithm not only solves the problem that particle swarm is easy to fall into local optimum, but also avoids the inappropriate initial value which leads to non-convergence of Newton iterative algorithm.

In the literature [26], the authors were focused on the NLOS node localization problem for WSN. Firstly, they use the modified Kalman Filter algorithm to reduce the NLOS error according to its distribution model. Moreover, combined with the least square method(LSM), the reconstructed measured value is used to estimate the general location of the target node. Finally, the higher localization accuracy can be obtained by applying the particle swarm optimization(PSO) algorithm.

In the literature [27], the paper proposed efficient localization algorithm that enables sensor nodes to estimate their location with high accuracy. The purpose of this paper is to develop the particle swarm optimization assisted Extended Kalman Filter (PSO-EKF) for Localization in WSN.

The above methods that were proposed in the literatures can improve the location accuracy of the unknown nodes' location in some extent, but most of the research were focus on the calculation of average hop distance, few scholars have been able to optimize the estimation coordinates of the unknown nodes as the focus of research. In addition, when the DV-Hop algorithm is optimized by PSO algorithm, when the number of iterations is increased to 80 times, the fitness function of PSO algorithm is still not stable. In this paper, we propose a new and improved DV-Hop localization algorithm which is based on QPSO algorithm. In the optimization of QPSO algorithm, the number of iterations is less, and then the adaptation value is almost stable.

The influences of signal fading model, distribution of nodes, anchor nodes density and network parameter error on location error rate, coverage and energy consumption in DV-Hop localization algorithm are very small, and the energy consumption is little, the location error rate can be reduced with the increase of node communication radius. However, the location error of DV-Hop algorithm still cannot meet the application of high accuracy, the error rate of this algorithm is still relatively high, so it is necessary to analyze the cause of errors in DV-Hop localization algorithm, thereby reducing the error rate, which can be used in more scenarios.

\subsection{QPSO Algorithm}

QPSO (Quantum behaved Particle Swarm Optimization) is based on PSO (Particle Swarm Optimization) algorithm. PSO algorithm is proposed by Eberhard and Kennedy in 1995, it is a stochastic search technique based on group. The number of iterations in PSO algorithm is more, and it cannot converge to global optimal value. The application of the algorithm is easier to guide the results to the local minimum in DV-Hop, and the process will spend a lot of time in searching smaller function value. This is the main disadvantage of PSO algorithm. In order to overcome the above shortcomings, it uses QPSO algorithm to improve the accuracy of the nodes' location in DV-Hop algorithm [28].

The principle of QPSO algorithm is as follows:

In QPSO algorithm, the particles have quantum behavior, the algorithm is starting from quantum mechanics, and it is based on DELTA trap. The formula of the particle position is obtained through Monte Carlo stochastic simulation method. The formula of the particle position is shown in formula (8).

$$
X(t)=P \pm \frac{L}{2} \ln \left(\frac{1}{u}\right)
$$

In formula $(8), X(t)$ is the particle position, $P$ is the best historical value of particles, $U$ is a random number between $[0,1] . \quad L(t+1)=2 * \beta \mid$ mbest $-X(t) \mid$, the formula of mbest is shown in formula $(9)$.

$$
\text { mbest }=\frac{\sum_{i=1}^{M} P_{i}}{M}=\left(\frac{\sum_{i=1}^{M} P_{i 1}}{M}, \frac{\sum_{i=1}^{M} P_{i 2}}{M}, \cdots, \frac{\sum_{i=1}^{M} P_{i D}}{M}\right)
$$

In formula (9), $\mathrm{M}$ is the number of particles, and $D$ is the dimension of the particle swarm space. $P_{d}=\phi^{*} P_{i d}+(1-\phi) P_{g d}$, in the formula $P_{d}, P_{i d}$ is the optimal location of particle $i$ for current search (individual optimum value). $P_{g d}$ is the optimal location (global optimum) that can be found in all particles. $\beta=\frac{1}{2} \times\left(T_{M A X}-T\right) / T_{M A X}, \varphi$ is the random number between $[0,1]$. From the above analysis, we can see that $\beta$ is the 
parameter that determines the position and convergence rate of particles, which is the contraction and expansion factor.

The particles in PSO algorithm have velocity characteristics. In the algorithm, the updating of the particle position is relatively linear. The particle behavior of quantum space in QPSO algorithm is similar to human intelligence behavior, and the uncertainty is large. These characteristics indicate that QPSO algorithm is very suitable for describing particles in intelligent swarm algorithm. In addition, each particle in the QPSO algorithm has a wide field of view, which can search the global optimal solution more comprehensively. The iterative updating of particles is not limited by the speed. Thus, the particle evolution formulas in QPSO algorithm are shown in formulas (10) and (11).

$$
\begin{aligned}
& p_{i d}(t)=\phi^{*} P_{i d}(t)+[1-\phi]^{*} P_{g d}(t) \quad \phi(t) \sim U(0,1) \\
& X_{i}(t+1)=p_{i d}(t) \pm \beta^{*}\left|\operatorname{mbest}(t)-X_{i}(t)\right|^{*} \ln \left[1 / u_{i}(t)\right] \\
& u_{i}(t) \sim U(0,1)
\end{aligned}
$$

The process of QPSO algorithm is as follows:

Step 1: Initialization parameters. $\mathrm{t}=0$, the particle position is initialized in the particle swarm. The individual optimum position of each particle is $P_{i d}(0)$ and $P_{i d}(0)=X_{i}(0)$. The termination condition of the program is set up.

Step 2: Calculate the best position of each particle according to formula (10).

Step 3: Execute step 4 to 7 of the process for each particle i. Step 4: According to the fitness function, the fitness value $P_{i d}(t)$ of particle $\mathrm{i}$ is calculated at $\mathrm{t}$ moment and compared with the fitness value $P_{i d}(t-1)$ at $\mathrm{t}-1$ moment. Finally, the optimal fitness value is selected and updated to $P_{i d}(t)$.

Step 5: Compare the fitness value of $P_{i d}(t)$ with $P_{g d}(t-1)$, the global optimum is selected and updated to $P_{g d}(t)$ as the global best location and fitness value.

Step 6: According to formulas (5) and (6), it calculates the latest location of the particle swarm.

Step 7: Is it satisfied the termination condition? Yes, the process returns to step (2). Otherwise, it returns to step 2.

\section{The Main Reason for Producing Error in DV-Hop}

\section{Location Sensing Algorithm}

The main reasons for producing error in DV-Hop location sensing algorithm are three aspects. One is caused by average hop distance. The second is caused by the method of calculating coordinates. The last one is caused by isolated nodes.

\subsection{The Error Caused by Average Hop Distance}

In DV-Hop algorithm, one of the reasons that affect the localization accuracy is the distance between the unknown node and anchor nodes. However the distance is obtained mainly according to the average hop distance, however it usually has error when using hop distance instead of the linear distance between nodes. Therefore, the average hop distance is the main factor that affecting the localization accuracy in DV-Hop. The distance is estimated as the product of the minimum hops and the average hop distance. The principal diagram of average hop distance error in DVHop algorithm is shown in figure 3.

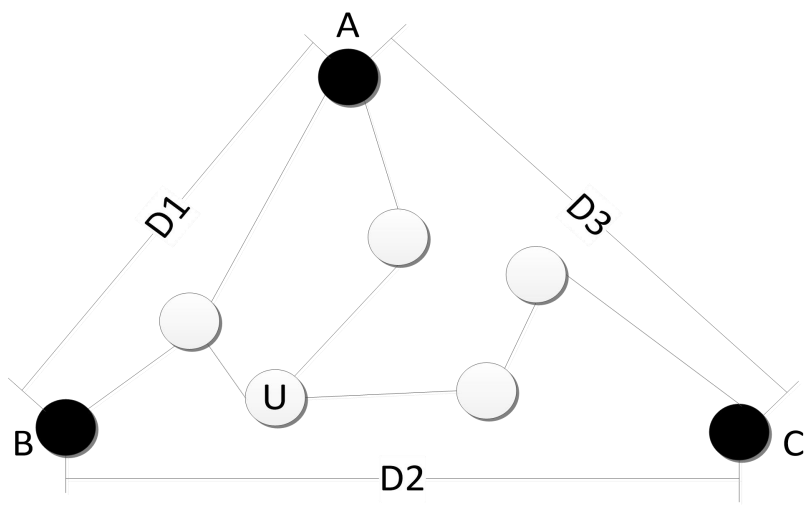

Fig 3. the principal diagram of average hop distance error in DV-Hop algorithm

Table 1. The Experimental Parameters (actual and estimation distance

\begin{tabular}{ll}
\multicolumn{2}{c}{ between anchor nodes) } \\
\hline \hline \multicolumn{1}{c}{ Experimental Parameters } & \multicolumn{1}{c}{ Parameter Values } \\
\hline Distribution & Square Random Distribution Region \\
Area & $200 \mathrm{~m} \times 100 \mathrm{~m}$ \\
Total Nodes & 120 \\
Unknown Nodes & 80 \\
Anchor Nodes & $0.02 / \mathrm{m} 2$ \\
Node Density & $40 \%$ \\
Proportion of Anchor & $0 \%$ \\
Nodes & $\mathbf{3 0 m}$ \\
GPS Error Rate & \\
Communication radius &
\end{tabular}

The following experiment will compare the estimation distance and the actual distance between the anchor nodes, and analyze the error trend between them. The experimental 
parameters are shown in table 1. The distribution of the wireless sensor network is the square area, and the nodes are deployed randomly, the area is $100 \mathrm{~m}^{*} 100 \mathrm{~m}$, the total number of nodes is 200 (of which the unknown nodes are 120 , the anchors are 80 ), the nodes density is $0.02 / \mathrm{m} 2$, the percentage of anchor nodes is $40 \%$, GPS error rate is $0 \%$, the communication radius of nodes is $30 \mathrm{~m}$.

In the experiment, the estimation distances between the anchor nodes are calculated by DV-Hop algorithm. The principle is that the number of hops between the anchor nodes are multiplied by the average hop distance. The comparison between the estimated distance and the actual distance is shown in figure 4. From the experimental results, it can be seen that the difference of tendency between the estimated distance and the actual distance. The distance is between an anchor node and other anchor nodes. The abscissa in the graph represents the actual distance between the anchor node and other anchor nodes, and the ordinate represents the difference between the estimated distance and the actual distance of an anchor node and other anchor nodes.

$h_{r i}$ is the actual distance between an anchor node and other anchor nodes. hei represents the estimated distance between an anchor node and other anchor nodes, and $i$ represents the serial number of nodes. $h_{\text {diff }}=h_{r i}-h_{e i}$ represents the difference between the actual distance and the estimated distance of the anchor node and the other anchor nodes. When the value of $h_{\text {diff }}$ is negative, it means that the estimated distance is less than the actual distance. When the value of $h_{\text {diff }}$ is positive, it means that the estimated distance is greater than the actual distance. The relationship between them is shown in figure 3. As can be seen from figure 3 , the majority of anchor nodes are located in the areas where the estimated distance is greater than the actual distance.

\subsection{The Error Caused by the Method of Calculating}

\section{Coordinates}

In DV-Hop algorithm, when the distance between the unknown node and the anchor node is obtained, three-sided measurement or maximum likelihood estimation method can be used to calculate the location coordinates of the unknown nodes. However, three-sided measurement method is sensitive to error, and the average hop distance also has error. So the distances between the unknown node and anchor nodes are inaccurate. Therefore, there is great error when calculating the nodes' coordinates using three-sided measurement method.

It uses maximum likelihood estimation method to calculate the coordinates of unknown nodes, although this method uses minimum variance to solve the problem that exists in three-sided measurement method, but the more number of the distance is used, it amounts to more errors. So the errors of maximum likelihood estimation method are also great.

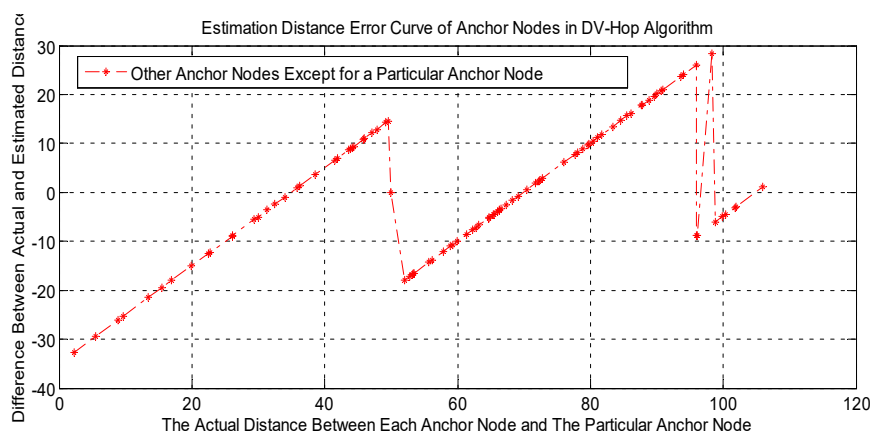

Fig 4. The Trend of Localization Error in DV-Hop Algorithm

\subsection{The Error Caused by Isolated Nodes}

In WSN, nodes are deployed randomly in most cases. It usually broadcasts the nodes to the region that to be tested through aircraft delivering, so there are usually some isolated nodes that can not to be located. This is also the main reason that affecting the error of unknown nodes locating.

In figure 5, it shows some isolated nodes in wireless sensor network. Figure 5(a) shows one isolated unknown node in wireless sensor network, the unknown node unable to communicate with any other nodes within its communication radius, so it is unable to locate. Figure 5 (b) shows the situation that there are two isolated nodes in wireless sensor network. Within the communication radius of the two isolated nodes there are no other nodes is unable to communicate with them, so it is unable to locate the two nodes. Figure 5(c) shows the situation of the resulting isolated nodes group in wireless sensor networks, in this case, within the communication ranges of these unknown 
isolated nodes there are few anchor nodes, and thus it is unable to locate them.

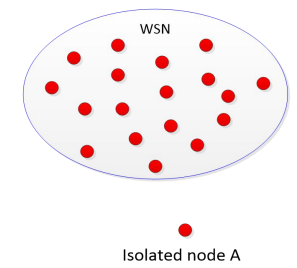

(a) One Isolated

Node

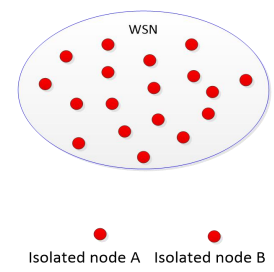

(b) Two Isolated

Node

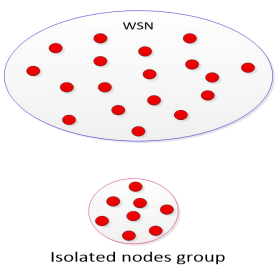

(c) Isolated
Fig 5. One Isolated Node Graph

\section{SQPSODV-Hop Localization Sensing Algorithm}

The two main reasons of localization error in DV-Hop localization sensing algorithm are the error of the average hop distance and calculating error of location coordinates. This section proposes a new localization sensing algorithm (SQPSODV-Hop) which improves these two aspects of DVHop localization sensing algorithm. Firstly, quantum behaved particle swarm optimization (PSO) is applied in DV-Hop algorithm, and then modifies the average hop distance in DV-Hop algorithm.

\subsection{The Application of QPSO Algorithm in DV-Hop}

In the DV-Hop localization algorithm, after using the three-sided measurement or maximum likelihood estimation method to calculate the location coordinates of the unknown nodes, QPSO algorithm is used to optimize the results. The specific optimization process is as follows:

(1) Parameter Initialization.

The population initialization of the unknown nodes estimated coordinates in wireless sensor networks, and then the parameters of the QPSO algorithm are initialized according to the basic particle swarm algorithm (PSO).

(2) Setting the fitness function. The fitness function value is set according to $V_{\text {Fitness }}$ in the PSO algorithm.

$$
V_{\text {Fitness }}=\sum_{i=1}^{N_{\text {anchor }}}\left(\sqrt{\left(x-x_{i}\right)+\left(y-y_{i}\right)}-d_{i}\right)^{2}
$$

(3) For each unknown node population, optimized the unknown nodes of each population according to QPSO algorithm.

(4) End the optimization of each node.
4.2 Optimization Average Hop Distance in DV-Hop Algorithm

QPSO algorithm revises coordinate values of the unknown nodes using adaptive value in DV-Hop algorithm. After revising it still cannot achieve zero error, the main reason is that the distances between the unknown nodes and anchor nodes are inaccurate, which is used to calculate the coordinates of unknown nodes. Therefore, even if the QPSO algorithm is used, the state of zero error cannot be reached. However, the greatest impact on the distances between unknown nodes and anchor nodes are the estimation of the average hop distance. Therefore, this section will correct the estimation of the average hop distance in DV-Hop algorithm. In DV-Hop algorithm, for a given anchor node, the process for calculating the average hop distance is as follows: first, calculating the distances between the anchor node and all other anchor nodes around it. Then calculating the sum of these distances. Last, dividing the total distance by the sum of hops. The average value is the average hop distance. From 3.1, we can see that the average hop distance is smaller for the anchor node closer to the anchor node and vice versa. Therefore, the law of this error can be used as a weighted value to correct the average hop distance.

In addition to consider the average hop distance of the nearest anchor node of the unknown node, it needs to ignore farther anchor node in the revising the average hop distance.

For each unknown node $i$, judging each node $j$ whether it is the nearest anchor node from the unknown nodes. If it is, the node $\mathrm{j}$ is taken as the target anchor node, then selecting each of the other anchor nodes circularly. Through the above process, if there is anchor node that can be satisfied the formula (13), the hop number between the anchor node and the target anchor node $\mathrm{j}$ is 1 , or the distance between the anchor node and the target node $j$ is less than the product of the WSN area and the density of the anchor node. In addition to consider the average hop distance of the nearest anchor node in the unknown node, the average hop distance of the farther anchor node is also ignored in the revision of the average hop distance.

$$
h_{j k}==1 \| d_{j-k} \leq \text { Area } *\left(2 * n_{\text {anchor }} /\left(5 * n_{\text {sum }}\right)\right)
$$


In formula (13), $h_{j k}$ represents the hop number between anchor node $\mathrm{k}$ and the target anchor node $j . d_{j-k}$ represents the distance between anchor node $k$ and the target anchor node $j$. Area is the area of the wireless sensor network. $n_{\text {anchor }}$ is the number of anchor nodes. $n_{\text {sum }}$ is the total number of the nodes. The difference value is calculated between the actual distance and the estimated distance from the anchor node and target anchor node which satisfied the formula (13). The difference value is shown in formula $(14)$.

$$
\operatorname{diff}_{j-k}=d_{R j-k}-d_{E j-k}
$$

$\operatorname{diff}_{j-k}$ is the difference value of target anchor node $\mathrm{j}$ and anchor node $\mathrm{k}$, and dividing this value by the actual distance $d_{r j-k}$ between the anchor node $\mathrm{k}$ and the target anchor node $\mathrm{j}$ as the correction value $P_{E h(j, k)}$ between them. $P_{E h(j, k)}$ is shown in formula (15).

$$
P_{E h(j, k)}=\operatorname{dhop}(j) *\left(1+\operatorname{diff}_{j-k}\right) / d_{R j-k}
$$

The new average hop distance is shown in formula (16). HopSize $_{\text {new-average }}$ is the new average hop distance.

$$
\text { HopSize }_{\text {new-average }}=\sum_{1 \leq k \leq n_{\text {sum }}} P_{E h(j, k)} / n_{\text {anchor }}
$$

\subsection{SQPSODV-Hop Algorithm}

SQPSODV-Hop algorithm is a location sensing algorithm that correcting the average hop distance and optimizing the coordinates of unknown nodes by using QPSO algorithm in DV-Hop algorithm. The algorithm improves the two main factors that affect the DV-Hop algorithm. The process of the SQPSODV-Hop algorithm is shown in figure 6.

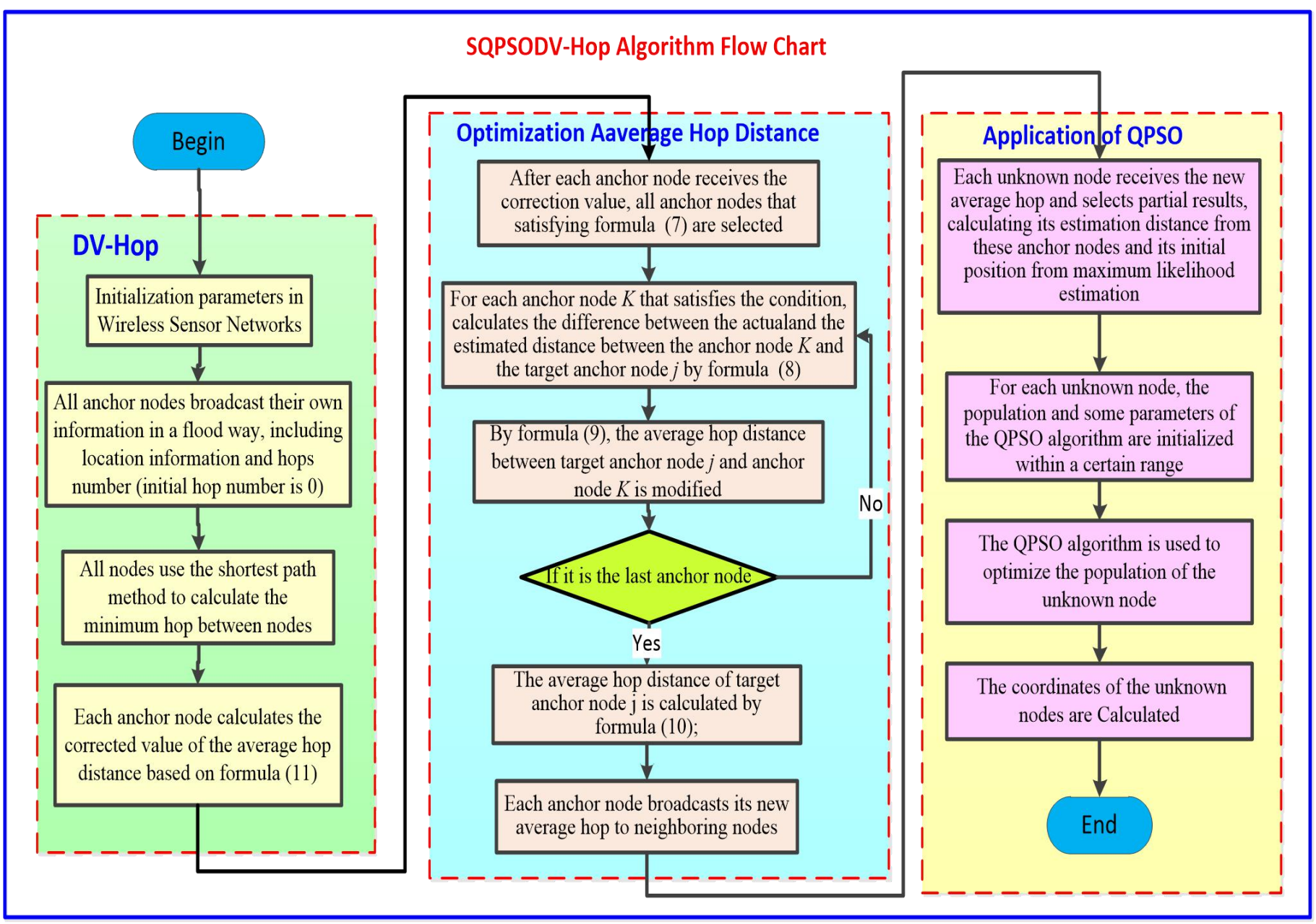

Fig 6. SQPSODV-Hop Algorithm Flow Chart 
(1) The parameters are initialized in Wireless Sensor Networks;

(2)All anchor nodes broadcast their own information in a flood way, including location information and hops number (initial hop number is 0 ).

(3) All nodes use the shortest path method to compute the minimum hop between nodes;

(4) Each anchor node calculates the corrected value of the average hop distance based on formula (17);

(5)After each anchor node receives the correction value, all anchor nodes that satisfying formula (13) are selected;

(6) For each anchor node $\mathrm{k}$ that satisfies the condition, calculating the difference between the actual and the estimated distance between the anchor node $\mathrm{k}$ and the target anchor node $\mathrm{j}$ by formula (14);

(7) By formula (15), the average hop distance between target anchor node $\mathrm{j}$ and anchor node $\mathrm{k}$ is modified;

(8) Determine whether it is the last anchor node; if so, enter the process (9), or else go to the process (6);

(9) The average hop distance of target anchor node $j$ is calculated by formula (16);

(10) Each anchor node broadcasts its new average hop to its neighboring nodes;

(11) Each unknown node receives the new average hop and selects partial results, calculating its estimation distance from these anchor nodes and its initial position from maximum likelihood estimation;

(12) For each unknown node, the population and some parameters of the QPSO algorithm are initialized within a certain range.

(13) The QPSO algorithm is used to optimize the population of the unknown node;

(14) End.

$$
\text { HopSize }_{i}=\frac{\sum_{i \neq j} \sqrt{\left(x_{i}-x_{j}\right)^{2}+\left(y_{i}-y_{j}\right)^{2}}}{\sum_{i \neq j} h_{i j}}
$$

5 Analysis of Communication Overhead and Computational Complexity in SQPSODV-HOP Algorithm
This section analyzes the two aspects of communication overhead and computational complexity in the SQPSODVHOP algorithm. In the algorithm, after calculating the anchor nodes' correction value, a new average hop distance is calculated according to the obtained hop distance values and flooding the new average hop distance information in SQPSODV-HOP algorithm. This process does not include communication between nodes, not increase additional communication overhead. In the optimization of the unknown node's estimated coordinate in DV-Hop algorithm, the communication between nodes is not involved in the process of the QPSO algorithm. Therefore, the application of QPSO algorithm in DV-Hop algorithm does not increase the communication overhead. In general, SQPSODV-HOP algorithm does not increase the communication overhead compare with DV-Hop algorithm.

The computation complexity of SQPSODV-HOP algorithm is mainly reflected in the computation of unknown nodes' coordinates and the implementation of QPSO algorithm. The implementation process of SQPSODV-HOP algorithm is a distributed parallel computing. The calculation complexity is the resources required to execute the algorithm (for example, time and storage capacity). It is usually represented by "O". For example, the algorithm requires $m$ inputs and the maximum number of basic operations is $3 m^{3}+5 m$, and the computational complexity of the algorithm is $O\left(\mathrm{~m}^{3}\right)$. For SQPSODV-HOP algorithm, the computation of unknown nodes' coordinates consists of three aspects. It is shown in formula (18).

$$
\left[\begin{array}{l}
x \\
y
\end{array}\right]=\left(A^{T} A\right)^{-1} A^{T} B
$$

In formula (18), the amount of computation of the matrix is required. It supposes that the number of anchor nodes is $n_{a n c}$, and the matrix $A$ is a $\left(n_{a n c}-1\right)$ rows and 2 columns, then $A^{T}$ is a matrix of 2 rows and $\left(n_{\text {anc }}-1\right)$ columns, $B$ is a matrix of $\left(n_{\text {anc }}-1\right)$ rows and 1 columns.

Since every element in the matrix $A$ requires one "-" and one " $x$ " operation, the basic operations in the matrix $A$ are $2\left(n_{a n c}-1\right)$ "-" operations and $2\left(n_{a n c}-1\right) " \times$ " operations. In the matrix $B$, the operations include "+", "-" and " $\times$ ", among 
these operations, the operation of " + " is $2\left(n_{a n c}-1\right)$, the operation of "-" is $3\left(n_{\text {anc }}-1\right)$, the operation of " $\times$ " is $3\left(n_{\text {anc }}-1\right) \cdot A^{T} A$ is the multiplication of two matrices $A$ and $A^{T}$, which demands $4\left(n_{a n c}-1\right) " \times$ " and $4\left(n_{a n c}-1\right) "+"$ $A^{T} A$ is a matrix of 2 rows and 2 columns, its inverse $\left(A^{T} A\right)^{-1}$ only demands one "-" operation, four " $\times$ " operations and four "/" operations. So $\left(A^{T} A\right)^{-1}$ and $A^{T}$ demand 2( $\left.n_{a n c}-1\right)$ "+" and 2( $\left.n_{a n c}-1\right)$ " $\times$ " operations. The matrix of $\left(A^{T} A\right)^{-1} A^{T} B$ demands $2\left(n_{a n c}-1\right) \quad " \times "$ and $2\left(n_{\text {anc }}-1\right) \quad$ "+" operations. Thus the computation of unknown nodes' coordinates demands $\left(10 n_{\text {anc }}-16\right)$ "+", $\left(5 n_{\text {anc }}-4\right)$ "-", $\left(15 n_{\text {anc }}-5\right)$ " × " and four "/" operations. Therefore, the computational complexity of SQPSODV-HOP algorithm is $O\left(n_{\text {anc }}\right)$.

In the algorithm, when the coordinate is optimized by QPSO algorithm, the time complexity is proportional to the product of the population number $M$ and the maximum number of iterations. Since the QPSO algorithm is used in the localization algorithm, the number of $M$ is not large, and the number of iterations is significantly reduced. As a consequence, the increases of the computational complexity is little. Most of the computation is equally distributed to each node, and the computation is not increased greatly.

Therefore, from the analysis of the computational complexity of the algorithm, the overall computational

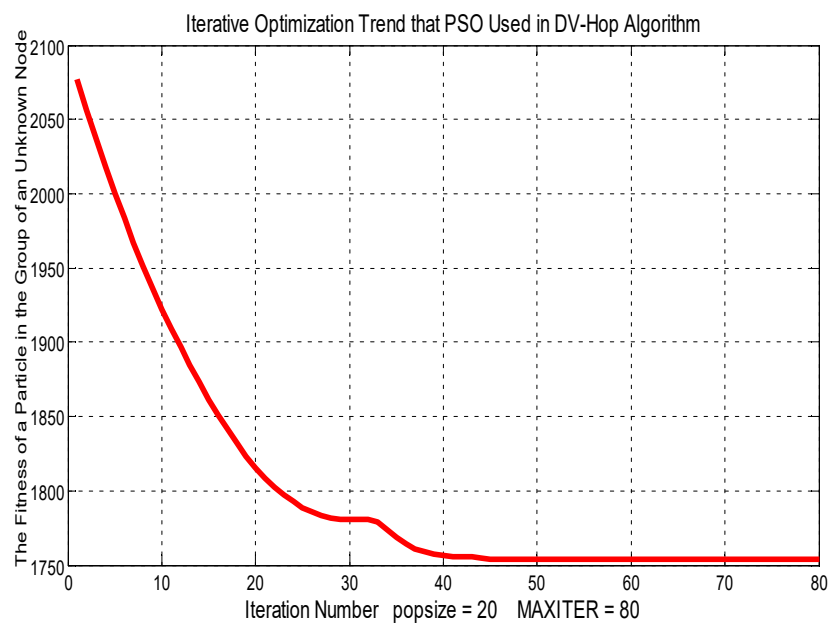

(a) complexity increases with the number of anchor nodes increases, and the QPSO algorithm is used in the algorithm, the population of $M$ is little, the iteration number is significantly reduced, so the computational complexity of the algorithm is increased slightly. Therefore, the algorithm reduces the localization error rate and increases the computational complexity.

\section{Iterative Trends Comparison of PSO and QPSO}

\section{Algorithm}

The following is the comparison of iterative trends between PSO and QPSO algorithm that used in DV-Hop algorithm.

Since the QPSO algorithm is based on the improvement of PSO algorithm, this part will compare the trend of iterative optimization in the two algorithms when optimizing the DV-Hop algorithm. In the optimization comparison of PSO and QPSO algorithm with the DV-Hop algorithm, it is the optimization comparison of the estimation coordinates of the same node in the DV-Hop algorithm. In the experiment, the area is $100 \mathrm{~m}^{*} 100 \mathrm{~m}$ and the total number of nodes is 100 , of which the anchor nodes are 40 , the communication radius is $30 \mathrm{~m}$. popsize represents the group size, MAXITER represents iteration number. The comparison results are shown in figures 7 and 8 . In figure 7 and 8 , the horizontal ordinate is the number of iterations, the vertical ordinate is the fitness of an unknown node's population when the two algorithms optimizing the results.

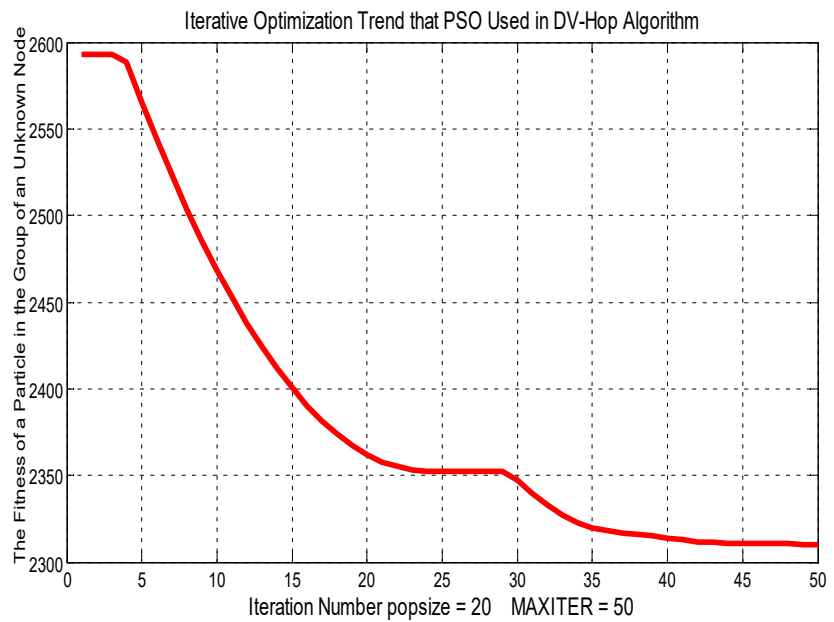

(b) 


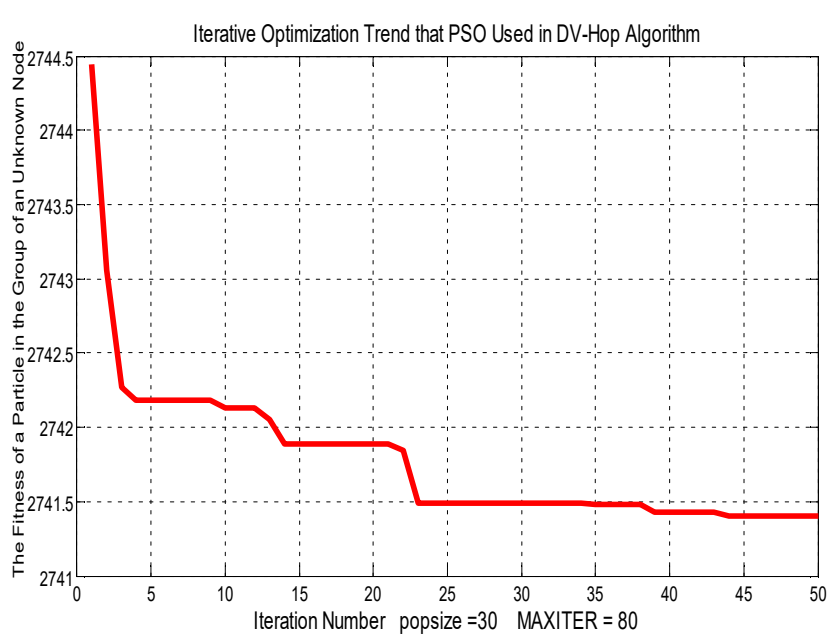

(c)

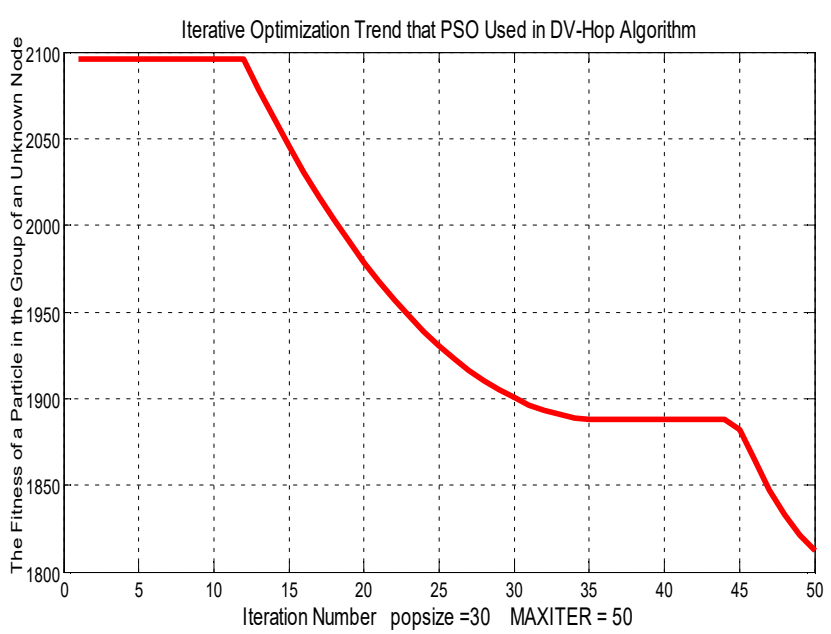

(d)

Fig 7. The Iterative Optimization Trend of PSO Application in DV-Hop Algorithm

In figure $7(\mathrm{a})$, popsize $=20$, MAXITER $=80 . \mathrm{DV}$-Hop algorithm is optimized by PSO algorithm, the number of iterations is up to 43 , and the fitness function is stable. In figure 7 (b), popsize $=20$, MAXITER $=50$. The number of iterations is up to 47 , the fitness function is stable. In figure 7 (c), popsize $=30$, MAXITER $=80$. The number of iterations is up to 48 , the fitness function is stable. In figure 7 (d), popsize $=30$, MAXITER $=50$. When the number of iterations is 50 , the fitness function is still not stable.

In figure 8, DV-Hop algorithm is optimized by QPSO algorithm. In figure 8 (a), popsize $=20$, MAXITER $=80$.

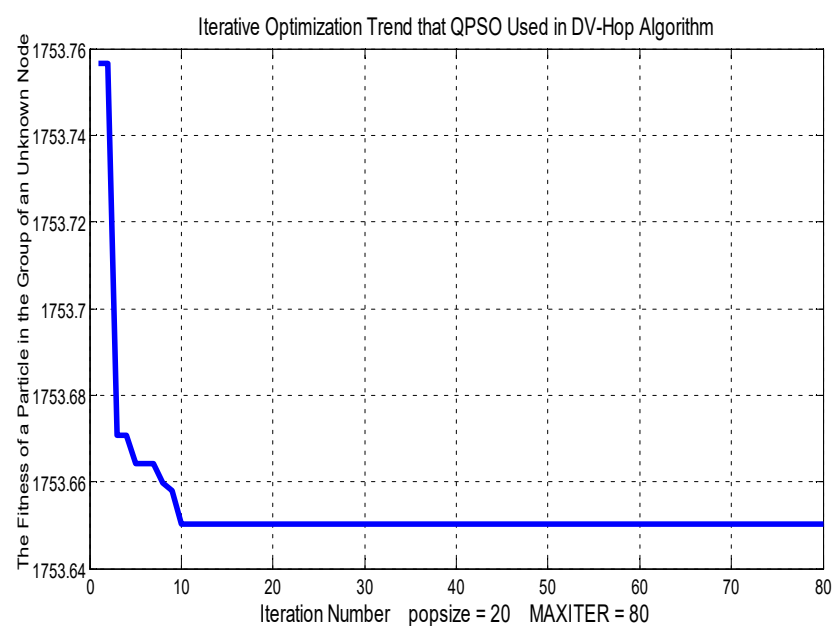

(a)
DV-Hop algorithm is optimized by PSO algorithm, the number of iterations is up to 10 , and the fitness function is stable. In figure 8 (b), popsize $=20, M A X I T E R=50$. The number of iterations is up to 9 , the fitness function is stable. In figure $8(\mathrm{c})$, popsize $=30, M A X I T E R=80$. The number of iterations is up to 5 , the fitness function is stable. In figure $8(\mathrm{~d})$, popsize $=30$, MAXITER $=50$. When the number of iterations is 7 , the fitness function is stable. In the optimization procedure of QPSO, after the iterative number of the group particle up to about 7 , the fitness is basically stable, and the function is globally convergent.

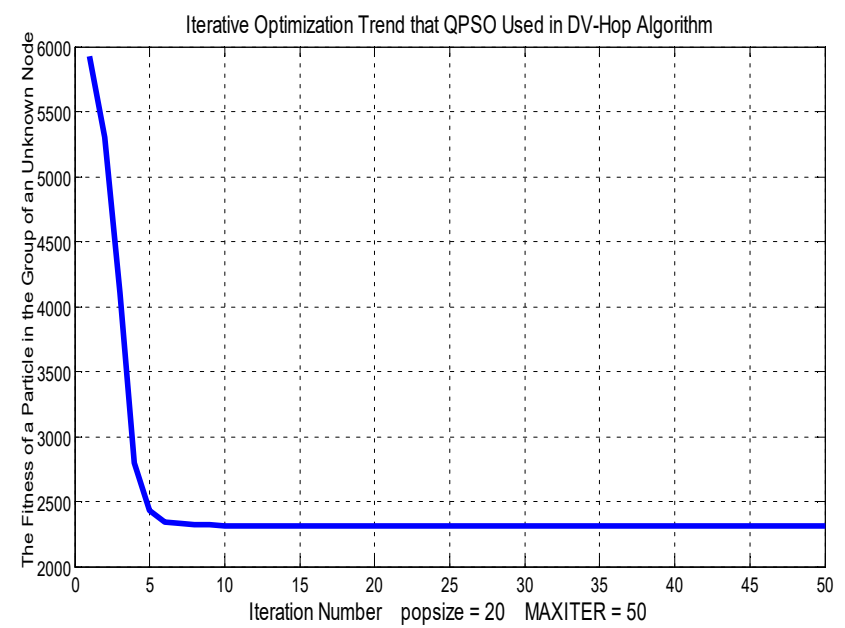

(b) 


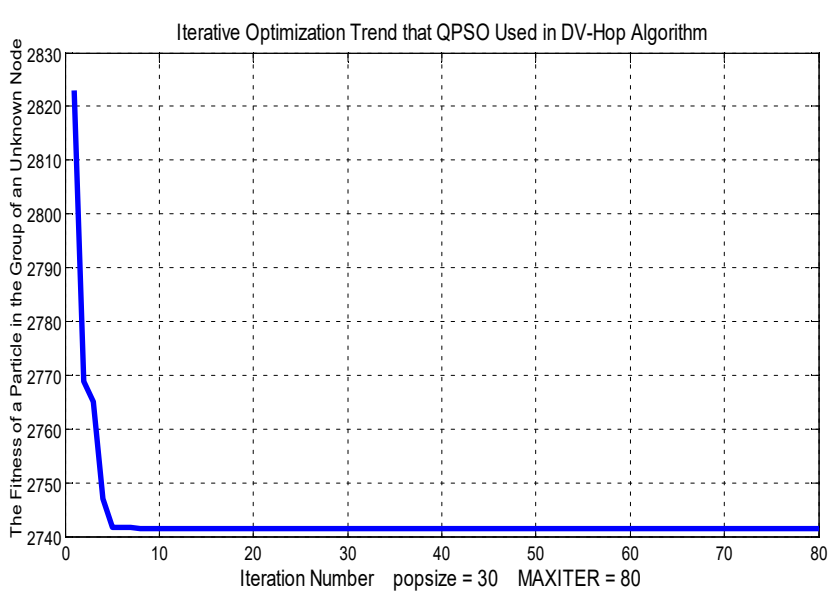

(c)

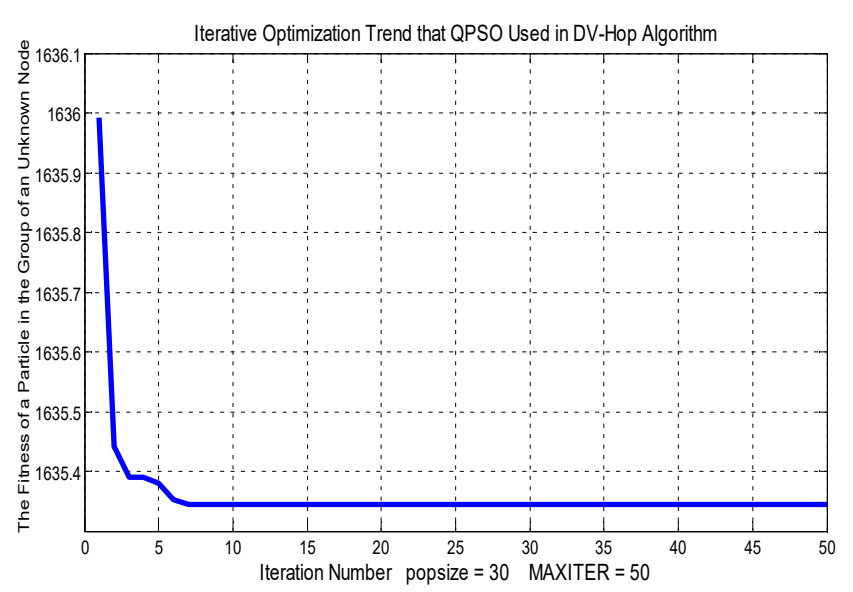

(d)

Fig 8. The iterative optimization trend of QPSO application in DV-Hop algorithm

Therefore, when the DV-Hop algorithm is optimized by PSO algorithm, the number of iterations is so many. When the number of iterations increases to 50 in figure $6(\mathrm{~d})$, the fitness function is still not stable. Therefore, the QPSO algorithm can reduce the computational burden greatly when optimizing the DV-Hop localization algorithm.

\section{Simulation Experiment and Results Analyze}

\subsection{Experimental Analysis of Fitness Function}

In this section, the experiments are carried out on the variation trend of the fitness function. The fitness function is that the PSO algorithm is applied in DV-Hop location sensing algorithm. In the initialization, it uses 400 sensor nodes as a population of particles, we choose one of the unknown nodes randomly in these nodes, bring it into the fitness function, and the coordinate of the unknown node is assumed to be known. The fitness function is shown in formula (11). The fitness function trend of the PSO algorithm is shown in figure 9. In the figure, the horizontal ordinate is the abscissa value of each node in the population, and the vertical ordinate is the fitness function value of each point in the population.

It can be seen from the figure, the PSO algorithm's fitness function is irregular, multimodal and complex. The algorithm is used in DV-Hop, and the results are easy to produce local minimum values, and the algorithm will take a lot of time in searching for smaller function values. Therefore, this part uses the QPSO algorithm to optimize the DV-Hop algorithm results, and the QPSO algorithm converges fast and global optimum. It plays an important role in the improvement of DV-Hop algorithm.

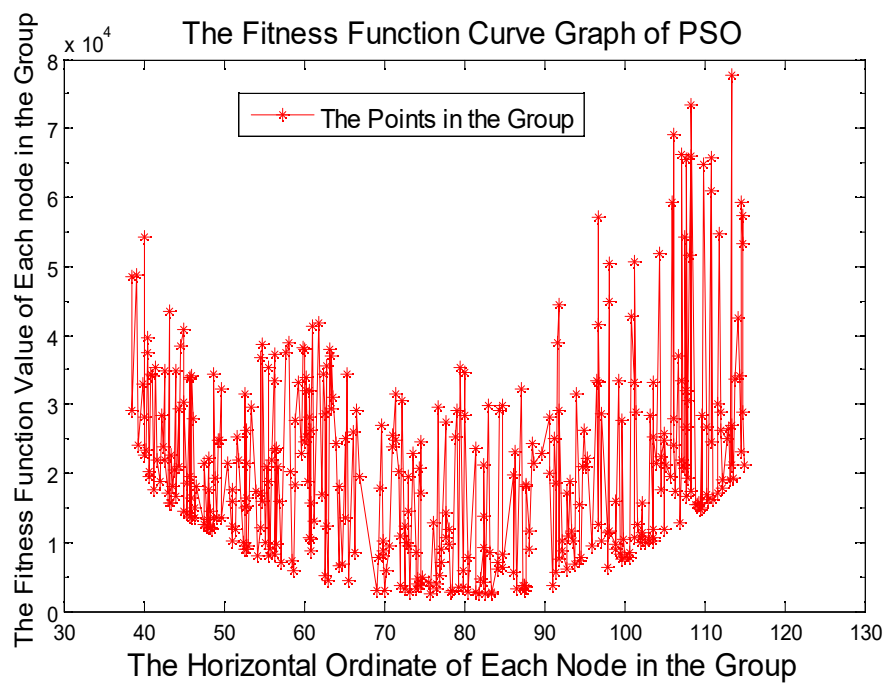

Fig 9. The Curve Graph of Fitness Function in PSO Algorithm

\subsection{Experiment and Results Analysis}

In order to analyze the performance of SQPSODV-Hop localization algorithm, this algorithm is compared with DVHop algorithm and PD-DVHop algorithm. The simulation software for this experiment is MATLAB R2012a, the operating system that running MATLAB R2012a is Windows 7, the processor is Intel Core i7-2630QM, $2.27 \mathrm{GHz}$, the memory is $4.00 \mathrm{~GB}$. The experiment is carried out in the area of $100 \mathrm{~m}^{*} 100 \mathrm{~m}$, and the communication radius and the number of anchor nodes vary according to the experimental requirements, and the nodes distribution is uniform. 
The experiment analyzes the average location error and the normalized average location error of the unknown nodes. The formula of average localization error is shown in (19). The formula of normalized average localization error is shown in (20). The localization error is tested through $e_{\text {average }}$ and $e_{\text {nor_average }}$. In formulas 19 and $20, x_{i}, y_{i}$ is the estimated coordinate of ith unknown node. $x_{a i}, y_{a i}$ are the actual coordinate of ith unknown node. $\mathrm{n}$ is the number of the unknown nodes. $\mathrm{R}$ is the communication radius of the nodes. The parameters include the number of anchor nodes, the density of nodes and the radius of communication. In the experiment, the comparison algorithms include DV-Hop algorithm, PD-DVHop and SQPSODV-Hop algorithm. In addition, the iterative convergence times of SQPSODV-Hop and PSODV-Hop will be analyzed and compared.

$$
\begin{aligned}
e_{\text {average }}= & \frac{\sum_{i=1}^{n} \sqrt{\left(x_{i}-x_{a i}\right)^{2}+\left(y_{i}-y_{a i}\right)^{2}}}{n} \\
e_{\text {nor_average }}= & \frac{\sum_{i=1}^{n} \sqrt{\left(x_{i}-x_{a i}\right)^{2}+\left(y_{i}-y_{a i}\right)^{2}}}{n R} \times 100 \%
\end{aligned}
$$

(1) Average Localization Error Varying Anchor Node Number

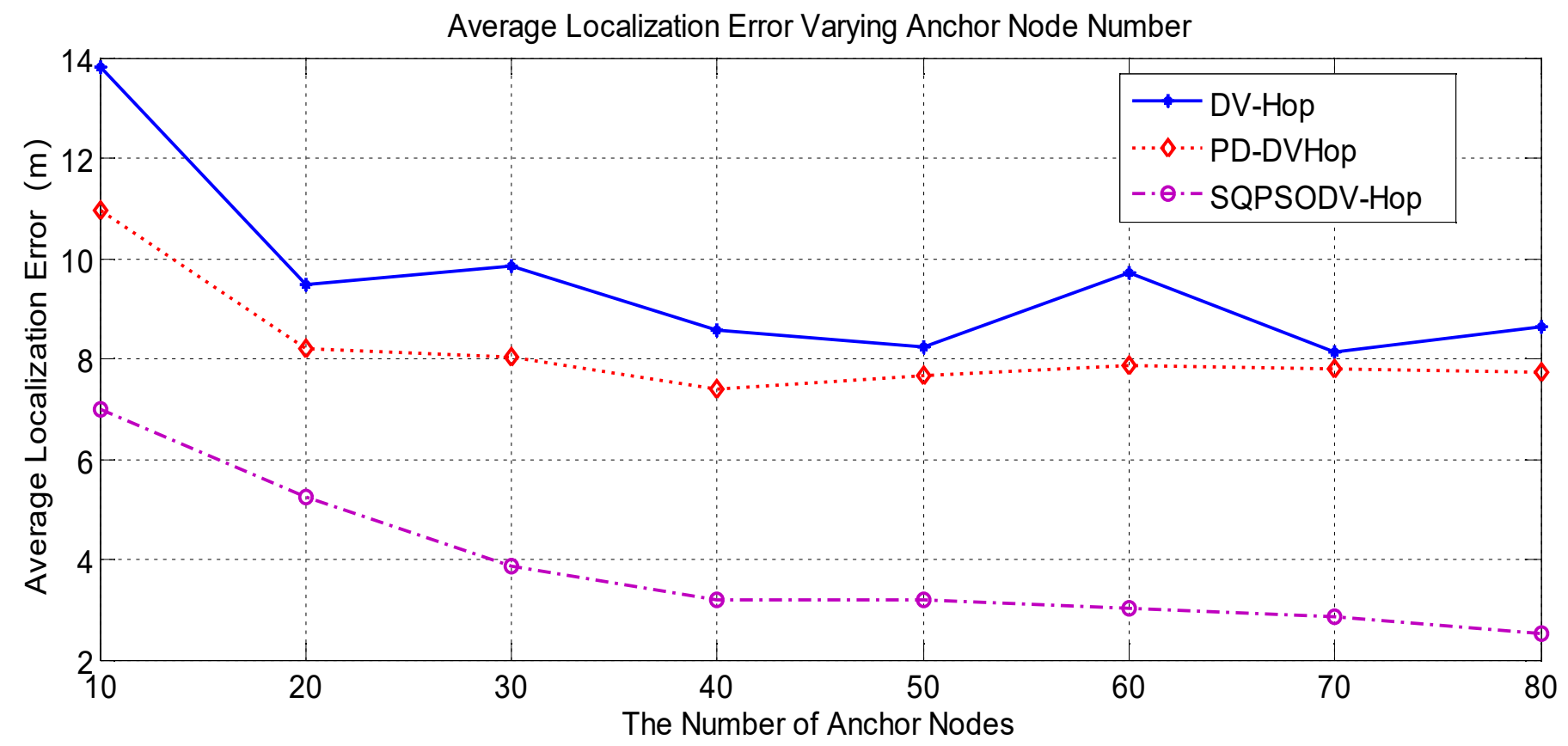

Fig 10. Average Localization Error Varying Anchor Node Number

In the area of $100 \mathrm{~m}^{*} 100 \mathrm{~m}$, the total number of the nodes is 200 , and the number of the anchor nodes is 10,20 , $30,40,50,60,70$ and 80 respectively. The communication radius of nodes is $30 \mathrm{~m}$. The maximum iteration number of PD-DVHop and SQPSODV-Hop algorithm is 20, and the population size is 20 . The simulation results of the average location error of different anchor nodes are shown in figure 10. As can be seen from figure 10, the average location error of the DV-Hop localization algorithm is the largest, followed by the PD-DVHop localization algorithm. The minimum localization error is the SQPSODV-Hop algorithm. With the increase of anchor nodes number, the average localization error of the SQPSODV-Hop algorithm decreases gradually, and decreases from $6.98 \mathrm{~m}$ to $2.53 \mathrm{~m}$. Thus, compared to the DV-Hop and PD-DVHop algorithms, the average localization error of the SQPSODV-Hop algorithm is the best.
(2) Normalized Average Localization Error Varying Communication Radius

In this experiment, the total number of the nodes is 100 , the number of the anchor nodes is 20 , the communication radius of nodes are $20 \mathrm{~m}, 25 \mathrm{~m}, 30 \mathrm{~m}, 35 \mathrm{~m}, 40 \mathrm{~m}, 45 \mathrm{~m}$ and $50 \mathrm{~m}$ respectively. The trend of normalized average location error of unknown nodes is analyzed and compared. The simulation results of the normalized average location error of different communication radius are shown in figure 11 . It can be seen from the figure, when the communication radius of nodes are increased from $20 \mathrm{~m}$ to $50 \mathrm{~m}$, the overall trend of the normalized average location error of the three algorithms is decreased, and when the communication radius is equal, the minimum normalized average location error of SQPSODV-Hop algorithm is $0.16 \mathrm{~m}$, it is better than DVHop and PD-DVHop algorithm. 


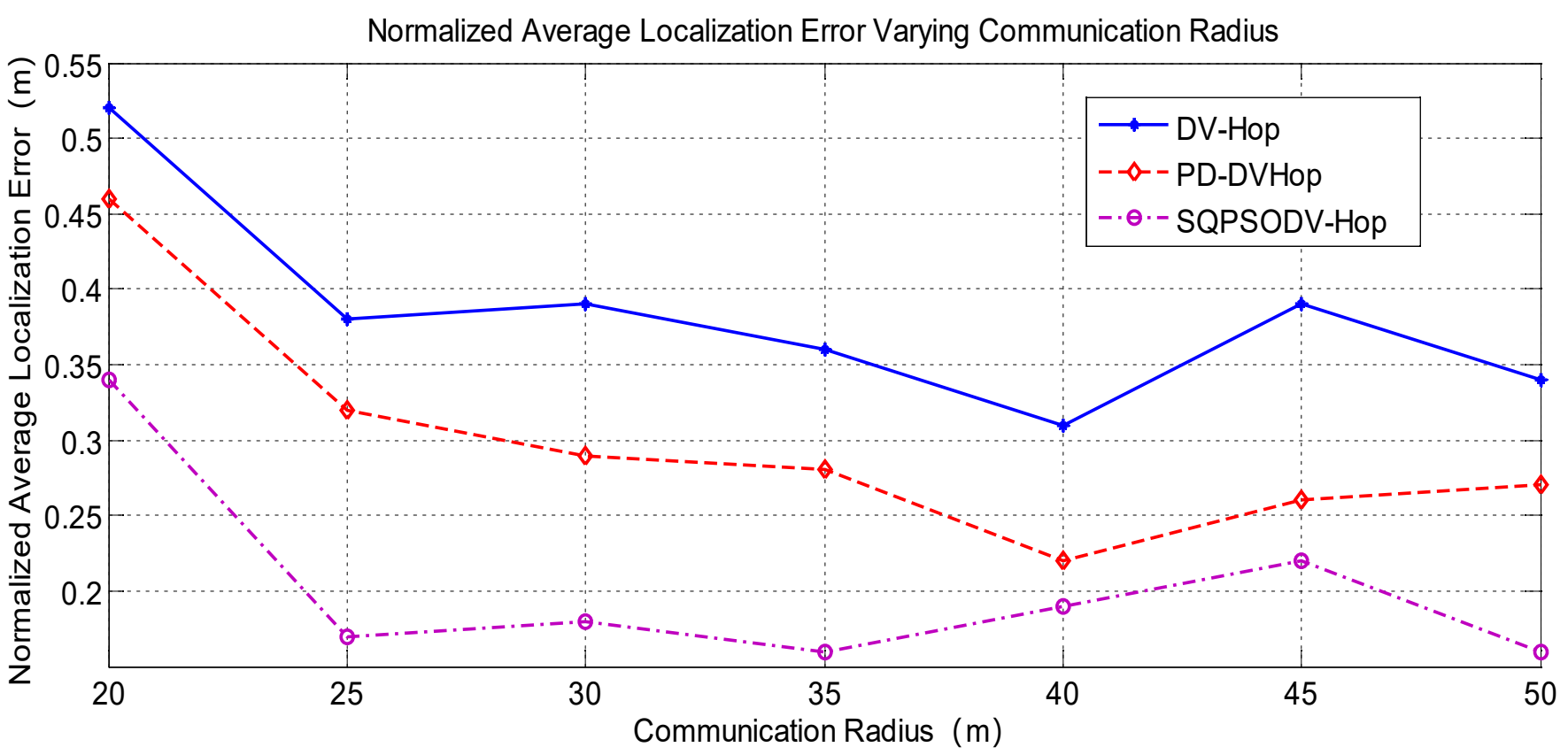

Figure 11. Normalized Average Localization Error Varying Communication Radius

(3) Normalized Average Localization Error Varying Nodes Density

The proportion of the anchor nodes and the communication radius are unchanged in this experiment, and the total number of nodes increases to analysis the normalized average location error of the unknown node. The anchor nodes density is $20 \%$ and the communication radius of nodes are $40 \mathrm{~m}$. The simulation results of normalized average location error at different nodes density are shown in figure 12. Where nodes density is the number of nodes per square meter. It can be seen from the figure, with the nodes density is increased, the overall normalized average location error of the three algorithms is decreased, and the performance of the SQPSODV-Hop algorithm is optimal, the normalized average location error is down to $0.1 \mathrm{~m}$ when the node density is 2.5 . PSO algorithm is used to optimize the estimated coordinates of unknown nodes in SQPSODV-Hop algorithm. Through the simulation experiment, the location errors of the SQPSODV-Hop algorithm, DV-Hop and PDDV-Hop are compared and analyzed. The results show that the error rate of PDDV-Hop is the lowest and the result is the best under the same experimental conditions. Finally, the computational complexity, communication overhead and iterative optimization trend of the algorithm are analyzed. The two single performance index optimization algorithms reduce the location error rate and little increase in computational complexity. In addition, the two algorithms do not increase the communication overhead. Moreover, compared with PDDV-Hop, SQPSODV-Hop algorithm can achieve global convergence without increasing communication overhead with few iterations.

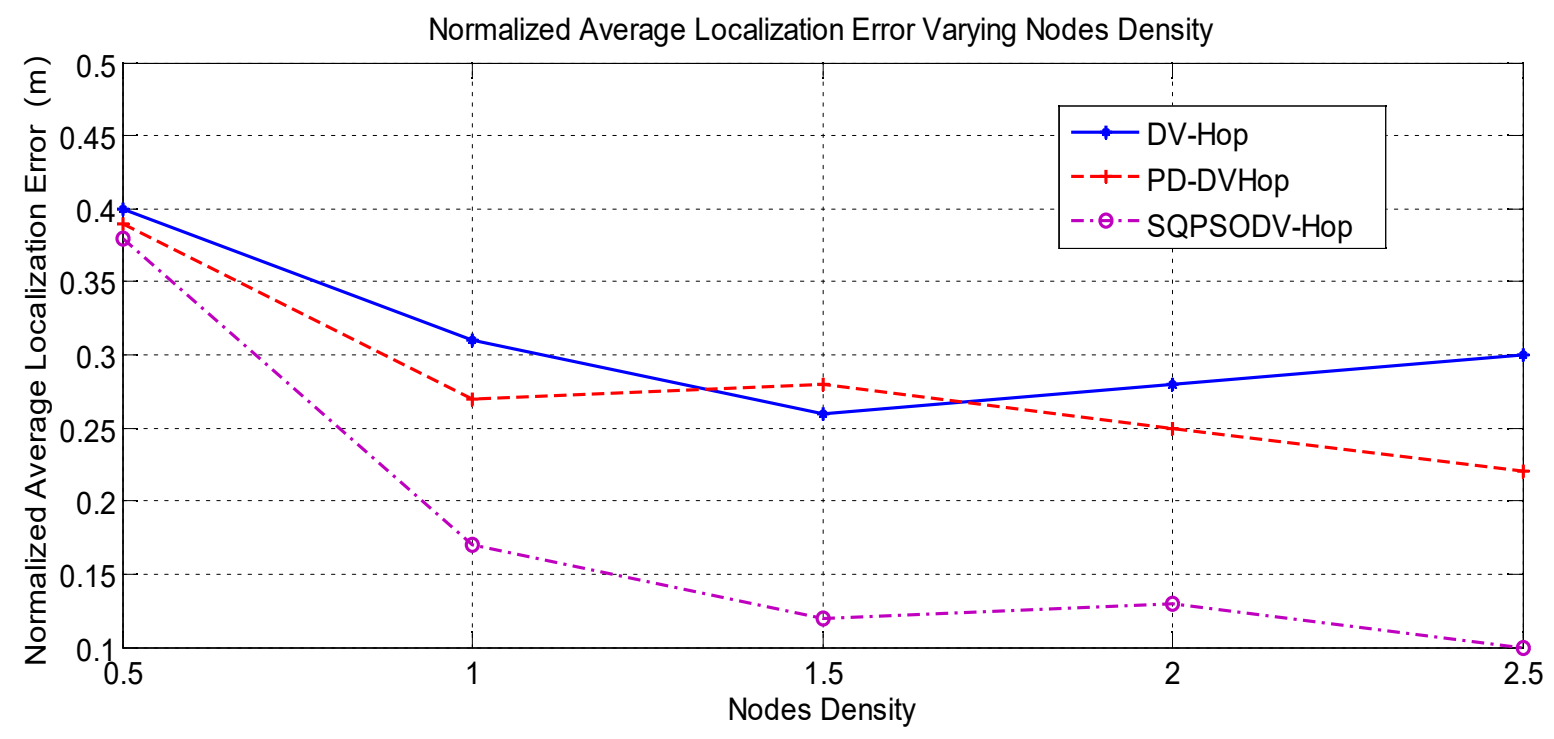

Fig 12. Normalized Average Localization Error Varying Nodes Density 


\section{Conclusion}

In this paper, the DV-Hop algorithm is improved on the basis of analyzing the factors that affecting the location accuracy of DV-Hop algorithm. It is mainly from the average hop distance correction and estimated coordinates optimization of the unknown nodes. In addition to considering the average hop distance from the nearest anchor node from the unknown node, the farther average hop distance from anchor nodes should be ignored. For the coordinates' estimation of unknown nodes, the optimization uses the QPSO algorithm. Finally, simulation experiments are carried out to compare the error of the new algorithm SQPSODV-Hop, DV-Hop algorithm and PDDV-Hop algorithm. The analysis results show that the performance of SQPSODV-Hop algorithm is the best under the same experimental conditions in the three algorithms.

\section{Acknowledgement}

This work was supported by the Professorial and Doctoral Scientific Research Foundation of Huizhou University under Grant 2018JB021.

\section{Reference}

[1] Pei Xie, Keyou You, Shiji Song, Cheng Wu. Distributed rangefree localization via hierarchical nonconvex constrained optimization[J].SignalProcessing,2019,164(0):136-145.

[2] Singh, M., \& Khilar, P. M. "Mobile beacon based range free localization method for wireless sensor networks,"Wireless Networks. 23 :1-16, 2016.

[3] Zhou, B., Jing, C., Kim, Y. "Joint TOA/AOA positioning scheme with IP-OFDM systems. In Proceedings of the IEEE INFOCOM," Toronto, ON, Canada, 27 April-2 May pp.1734-1743, 2014.

[4] R. Peng and M. L. Sichitiu. "Ad Hoc Positioning System (APS) using AoA," Twenty-Second Annual Joint Conference of the IEEE Computer and Communications, 3(2):1734-1743, 2003.

[5] Gaurav Sharma,Ashok Kumar.Modified Energy-Efficient Range-Free Localization Using Teaching-Learning-Based Optimization for Wireless Sensor Networks[J].IETE Journal of Research,2018,64(1):124-138.

[6] Munesh Singh,Pabitra Mohan Khilar, "A Range Free Geometric Technique for Localization of Wireless Sensor Network (WSN) Based on Controlled Communication
Range,” Wireless Personal Communications, Volume 94, Issue 3, 1359-1385, June 2017.

[7] Seyed Saber Banihashemian, Fazlollah Adibnia,Mehdi A.Sarram.A New Range-Free and Storage-Efficient Localization Algorithm Using Neural Networks in Wireless Sensor Networks $[\mathrm{J}]$.Wireless

Personal Communications,2018,98(1):1547-1568.

[8] Ademuwagun, A, Fabio, V. "Reach Centroid Localization Algorithm," Wireless Sensor Network. 87-101, 2017, 9.

[9] Feng, X.L., Xu, H.Y., Li, W., Sun, Z.Y,” Centroid position algorithm of structure optical stripe in asphalt pavement test," J. Optoelectron. Laser pp. 514-520, 2014, 3.

[10] Kaur, R., Malhotra, J. "Comparitive Analysis of DV-Hop and APIT Localization Techniques in WSN,” Int. J. Future Gener. Commun. Netw. 327-344, 2016, 9.

[11] Liu, J., Wang, Z., Yao, M., Qiu, Z. "VN-APIT: Virtual nodesbased range-free APIT localization scheme for WSN," Wirel, Netw. 867-878, 2016, 22.

[12] Sharma, R., Malhotra, S. "Approximate Point in Triangulation (APIT) based Localization Algorithm in Wireless Sensor Network,” Int. J. Innov. Res. Sci. Technol. 3942, 2015, 2.

[13] Zhang, Shi, Er, Meng Joo, Zhang, Baihai. "A novel heuristic algorithm for node localization in anisotropic wireless sensor networks with holes," SIGNAL PROCESSING, 138:27-34, 2017.

[14] Hongyu Sun, Zhiyi Fang. “CESILA: Communication circle external square intersection-based WSN localization algorithm. Sensors ef Transducers," 158(11): 1-8, 2013.

[15] D. Niculescu and B. Nath, "Ad hoc positioning system (APS),"in Proceedings of the IEEE Global Telecommunications Conference (GLOBECOM '01), vol. 5, San Antonio, Tex, USA, December 2001, pp. 2926-2931.

[16] S. Chen, R. Shu, S. Zhang, and X. Zheng, "Evaluate distance measurement how to influence localization in wireless sensor networks," in 2012 2nd International Conference on Consumer Electronics, Communications and Networks (CECNet), 323-325, 2012.

[17] Dan Zhang, Zhi-Yi Fang, Hong-Yu Sun,Jie Cao. "HTCRL: A Range Free Location Algorithm Based on Homothetic Triangle Cyclic Refinement in Wireless Sensor Networks," Information, 8(2):1-16, 2017.

[18] X. Zhang, T. Wang, and J. Fang, "A Node Localization Approach Using Particle Swarm Optimization in Wireless Sensor Networks," in 2014 International Conference on Identification, Information and Knowledge in the Internet of Things, 84-87, no. 31101080, 2014. 
[19] Su Bing and Xue Wei Jie. An Improved Algorithm Based on Dv-Hop Localization for Wireless Sensor Network [J]. Engineering and Applications, 2015, 220(9):181-190.

[20] Yuxiao Cao,Zhen Wang.Improved DV-Hop Localization Algorithm Based on Dynamic Anchor Node Set for Wireless Sensor Networks[J].Access, IEEE,2019,7 (0):124876-124890.

[21] Sana Messous,Hend Liouane,Noureddine Liouane.Improvement of DV-Hop localization algorithm for randomly deployed wireless sensor networks[J].Telecommunication Systems,2020,73(1):75-86.

[22] Yunzhou Zhang, Shu Xiang and Wenyan Fu, and Dongfei Wei. Improved Normalized Collinearity DV-Hop Algorithm for Node Localization in Wireless Sensor Network [J]. International Journal of Distributed Sensor Networks Volume 2014, 1-14.

[23] He Tian, Huang Chengdu, Blum B M, et al. Range-free localization schemes for large scale sensor networks [C]. The 9th Annual International Conference on Mobile Computing and Networking, ACM Press, 2005, 81- 95.

[24] S. Hou, X. Zhou, and X. Liu, "A novel DV-hop localization algorithm for asymmetry distributed wireless sensor networks," 3rd IEEE International Conference on Computer Science and Information Technology (ICCSIT), vol. 4, pp. 243248, 2010.

[25] Gao Li-Peng, Sun Heng, Liu, Meng-Nan, Jiang Yi-Lin. "TDOA collaborative localization algorithm based on PSO and Newton iteration in WGS-84 coordinate system," IEEE International Conference on Signal Processing , 1571-1575, 2017.3.

[26] Santar Pal Singh,Subhash Chander Sharma.Implementation of a PSO Based Improved Localization Algorithm for Wireless Sensor Networks[J].IETE Journal of Research,2019,65(4):502-514.

[27] Santar Pal Singh1;S. C. Sharma1.A PSO Based Improved Localization Algorithm for Wireless Sensor Network[J].Wireless Personal Communications, 2018,98(1):487-503.

[28] Lei Chen,Jiangfeng Wang,Jian Wang,Zhijun Gao,Jiakuan Dong,Xuedong Yan.A Wireless Localization Algorithm With BPNN-MEA-QPSO for Connected Vehicle[J].IEEE Intelligent Transportation Systems Magazine. (M-ITS),2019,11(2):96109.

\section{Biographies}

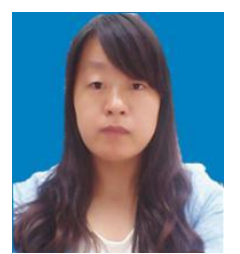

Dan Zhang (zhangdan@hzu.edu.cn) received the B.E. degree in electronic information engineering from the School of Information and Computer Engineering, Northeast Forestry University, Harbin, China, in 2003, and the Ph.D degree in computer system architecture from the School of Computer Science and Technology, Jilin University, Jilin, China, in 2017. She is currently a Professor with the School of Computer Science and Technology in Huizhou University. Her research interests include positioning and routing of wireless sensor networks, underwater robotics, computer vision.

Xiaohuan Zhang (zhangxiaohuan@hzu.edu.cn) was born in December 1983. She received her Ph.D. degree from Computer science and Technology, Harbin University of science and technology, Harbin, China in 2018. Currently, she is an lecturer in School of Computer Science and Engineering, Huizhou University. Her research interests include enterprise intelligent computing,algorithm analysis.

Hai Qi (Qh@hzu.edu.cn) was born in September 1978. He received a bachelor's degree. He graduated from network engineering of Harbin Institute of technology in 2007 and is now an engineer of network and information center of Huizhou University. Her research interests include network engineering, network security and intelligent computing. 